\title{
Ángulo de fase como indicador de riesgo cardiovascular en estudiantes universitarios
}

\author{
Phase angle as an indicator of cardiovascular risk in university students \\ Cristian J. Ramírez-Herrera, Juan C. Pico-Tarazona, Isabel A. Sánchez*, Felipe R. Garavito-Peña, \\ Darío Mendoza-Romero y Laura E. Castro-Jiménez \\ Facultad de Cultura Física, Deporte y Recreación, Universidad Santo Tomás, Bogotá, Colombia
}

\section{Resumen}

Introducción: El ángulo de fase se utiliza actualmente como indicador del estado nutricional de los adultos y marcador pronóstico de presencia y progresión de enfermedades crónicas, como las cardiovasculares. Objetivo: Determinar la asociación entre el ángulo de fase y los indicadores de riesgo cardiovascular en estudiantes universitarios. Método: Estudio correlacional de corte transversal, en el que se evalúo a 30 estudiantes universitarios (edad $22.1 \pm 2$ años, peso 65.6 $\pm 10,3 \mathrm{~kg}$ ) a través de IPAQ (versión corta), glucometría basal, composición corporal mediante bioimpedancia eléctrica con el instrumento Inbody® de referencia 770, fuerza prensil, batería de Bosco (Optogait $($ ) y consumo de oxígeno indirecto (test de Leger). Resultados: Se encontró una media de ángulo de fase de $6.4 \pm 0.66$, y se halló correlación moderada entre masa magra en tronco (0.68; $p$ $=0.05)$, tasa metabólica basal (0.64; $p=0.009)$, nivel de fitness $(0.71 ; p=0.003)$, Counter Movement Jump (0.56; $p=0.028)$ y ángulo de fase. Las mujeres presentan correlación entre relación de cintura y cadera $(r=0.74 ; p=0.034)$. Conclusiones: El ángulo demostró ser un indicador predictor de riesgo cardiovascular en población adulta joven; además, permitió una visión más exacta de la predisposición y la potencialidad para padecer enfermedad cardiovascular.

Palabras clave: Factores de riesgo. Composición corporal. Adulto joven.

\section{Abstract}

Introduction: The phase angle is currently used as an indicator of the nutritional status of adults and a prognostic marker of the presence and progression of chronic diseases such as cardiovascular diseases. Objective: To determine the association between phase angle and cardiovascular risk indicators in university students. Method: Correlational cross-sectional study. Thirty university students (age $22.1 \pm 2$ years, weight $65.6 \pm 10.3 \mathrm{~kg}$ ) were evaluated through IPAQ (short version), baseline glucometry, body composition using electrical bioimpedance with the Inbody $\otimes$ reference instrument 770 , prehensile force, Bosco battery (Optogait@), indirect oxygen consumption (Leger test). Results: $A$ mean phase angle $6.4 \pm 0.66$ was found, with a moderate correlation between lean trunk mass (0.68; $p=0.05)$, basal metabolic rate $(0.64 ; p=0.009)$, fitness level $(0.71 ; p=0.003)$, Counter Movement Jump (0.56; $p=0.028)$ and phase angle. Women have a correlation between waist and hip ratio ( $r=0.74 ; p=0.034)$. Conclusions: The angle proved to be a predictive indicator of cardiovascular risk in young adult population, also allowed a more accurate view of the predisposition and potential for cardiovascular disease.

Key words: Risk factors. Body composition. Young adult.

\section{Correspondencia:}

*Isabel A. Sánchez

E-mail: isabel.sanchez@usantotomas.edu.co
Disponible en internet: 07-01-2022 Rev Colomb Cardiol. 2021;28(6):656-664 www.rccardiologia.com 0120-5633 / @ 2020 Sociedad Colombiana de Cardiología y Cirugía Cardiovascular. Publicado por Permanyer. Este es un artículo open access bajo la licencia CC BY-NC-ND (http://creativecommons.org/licenses/by-nc-nd/4.0/). 


\section{Introducción}

Con el transcurso del tiempo han sido diversas las estrategias que se han implementado con el fin de predecir el riesgo cardiovascular; gran parte de estas han surgido a partir del estudio Framingham, el cual ha sido implementado para estimar el riesgo cardiovascular con base en variables específicas, como el padecimiento de enfermedades coronarias, el tabaquismo, la obesidad, el ejercicio físico, la dieta y el consumo de alcohol ${ }^{1}$. Sin embargo, existen múltiples investigaciones que se han basado en el análisis de la composición corporal y la condición física, como variables fisiológicas que se correlacionan de manera importante con el potencial de padecer enfermedades cardiovasculares.

La bioimpedancia eléctrica es un método de evaluación simple, rápido y no invasivo que permite determinar múltiples componentes, como el porcentaje de agua, la masa libre de grasa y la masa magra, entre otros, mediante estimaciones basadas en la hidratación de los tejidos ${ }^{2}$. Este método tiene como base fundamental las propiedades eléctricas del cuerpo, composición, maduración, estado de hidratación, edad, sexo y condición física de los individuos ${ }^{2,3}$.

La impedancia corporal (Z), la cual se mide en $\mathrm{Ohms}^{4}$, está en función de dos vectores fundamentales, que son la resistencia $(\mathrm{R})$ y la reactancia $(\mathrm{Xc})$, entendida como la oposición a una corriente eléctrica, manifestando así que $\mathrm{Z}^{2}=\mathrm{R}^{2}+\mathrm{Xc}^{2}$, en donde $\mathrm{R}$ es la resistencia de los tejidos al paso de una corriente eléctrica y Xc representa la oposición adicional de la capacitancia (capacidad de la membrana celular para mantener una carga eléctrica) en los tejidos ${ }^{2-5}$.

Este paso de corriente está definido por dos ondas que poseen distinta amplitud, pero una misma frecuencia, las cuales son la onda de intensidad y la onda de voltaje $^{4}$. Si se determina que los picos de las ondas coinciden, se establece que la intensidad está en fase con el voltaje, mientras que si existe un retraso de la onda de voltaje (ocasionada por la carga y descarga de los condensadores) respecto a la onda de intensidad se establece que la corriente está desfasada ${ }^{4}$; este desfase se denomina ángulo de fase.

El ángulo de fase es una medida calculada por la bioimpedancia eléctrica, que determina de manera indirecta la estabilidad celular ${ }^{6}$. Esta medida analiza la composición muscular estableciendo la cantidad de agua y electrolitos como indicadores del tamaño, el número y la integridad celular, la distribución de agua en los espacios intracelulares y extracelulares, indicando la integridad de la membrana celular y siendo un predictor de la masa celular corporal ${ }^{6}$. El ángulo de fase puede ser estimado mediante la ecuación $(\mathrm{Xc} / \mathrm{R}){ }^{*} 180 \%$, con la que se demuestra que el ángulo de fase es dependiente de la capacitancia de los tejidos, su celularidad, y el tamaño y la integridad de la membrana, como también es dependiente del comportamiento de la $\mathrm{R}$, que está relacionada con la hidratación de los tejidos?.

A partir de lo anterior, se establece que el ángulo de fase puede ser un indicador del estado nutricional en adultos y niños, como también un marcador pronóstico de condiciones clínicas y monitorización en la progresión de enfermedades ${ }^{6-8}$; en consecuencia, se han determinado valores de referencia para el ángulo de fase en población sana en rangos de $5-7^{\circ 8}$, en cuyo caso, los estudios han reflejado que los valores han sido de $5,3^{\circ}-7,8^{\circ}$ para los hombres y de $5,3^{\circ}-7,3^{\circ}$ para las mujeres ${ }^{6}$.

Este ángulo de fase presenta tres indicadores importantes mediante los cuales se podría predecir la presencia de factores de riesgo cardiovascular. El primero de ellos es la edad; se afirma que, a mayor edad, el ángulo de fase evidencia menor reactancia debido a la disminución de la masa magra, la oposición que inducen los tejidos biológicos que actúan como condensadores promotores de sus propiedades dieléctricas, la depleción del agua corporal y el incremento de la masa grasa ${ }^{7}$. Como segundo indicador, se encontró que los hombres, al poseer mayor cantidad de masa celular, tendrán un mayor ángulo de fase en comparación con las mujeres. En cuanto al índice de masa corporal (IMC), se evidencia que el ángulo de fase incrementa a medida que lo hace el IMC, debido al aumento de masa celular y muscular, demostrada solo en valores de normalidad o inferiores a $34 \mathrm{~kg} / \mathrm{t}^{2}$, caso en el cual el individuo tendrá un mayor ángulo de fase; por el contrario, si el sujeto tiene obesidad o un IMC superior a $40 \mathrm{~kg} / \mathrm{t}^{2}$, el ángulo de fase presenta menor reactancia y por lo tanto tendrá un efecto negativo ${ }^{8}$.

En virtud de lo anterior, el objetivo de este estudio es determinar la asociación entre el ángulo de fase y los indicadores de riesgo cardiovascular, con el fin de que la bioimpedancia eléctrica sea una herramienta que permita establecer el riesgo cardiovascular en población universitaria. Igualmente, se busca que los resultados y el análisis del proyecto abran la puerta de invitación a la generación de futuras investigaciones que lo implementen en otras poblaciones de estudio. 
Tabla 1. Descriptivos de la población $(n=30)$

\begin{tabular}{|c|c|c|c|c|}
\hline Variables & Mínimo & Máximo & Media & Desviación típica \\
\hline Edad (años) & 20.0 & 28.0 & 22.1 & 2.0 \\
\hline Peso (kg) & 35.9 & 86.3 & 65.6 & 10.3 \\
\hline Índice de masa corporal $\left(\mathrm{kg} / \mathrm{m}^{2}\right)$ & 19.7 & 30.2 & 23.4 & 2.9 \\
\hline Relación cintura-cadera (cm) & 0.79 & 0.98 & 0.84 & 0.04 \\
\hline Leger $\mathrm{VO}_{2 \text { máx }}(\mathrm{ml} / \mathrm{kg} / \mathrm{min})$ & 26.5 & 53.6 & 39.5 & 7.1 \\
\hline Squat Jump, altura (cm) & 17.5 & 42.4 & 27.9 & 7.0 \\
\hline Counter Movement Jump, altura (cm) & 18.1 & 45.9 & 30.6 & 7.5 \\
\hline Fuerza prensil izquierda & 18.0 & 51.5 & 33.9 & 9.5 \\
\hline Fuerza prensil derecha & 17.0 & 54.0 & 35.6 & 8.8 \\
\hline Total kilocalorías & 41.9 & 3284.2 & 1071.7 & 824.5 \\
\hline Tasa metabólica basal (kcal) & 1175.0 & 1910.0 & 1513.0 & 200.4 \\
\hline Glucometría (mg/dl) & 64.0 & 103.0 & 81.3 & 9.8 \\
\hline Ángulo de fase & 4.7 & 7.6 & 6.4 & 0.66 \\
\hline Nivel de fitness & 67.0 & 91.0 & 77.0 & 6.7 \\
\hline
\end{tabular}

\section{Método}

\section{Diseño del estudio}

Este estudio cuenta con un enfoque cuantitativo de tipo no experimental correlacional de corte trasversal, a fin de establecer la correlación existente entre el ángulo de fase con variables de composición corporal, condición física y nivel de actividad física. La población fue seleccionada por conveniencia; el promedio de edad fue de 22.1 años y el de peso fue de $65.5 \mathrm{~kg}$ (Tabla 1).

La muestra del estudio estuvo constituida por 30 participantes (22 hombres y 8 mujeres). Como criterios de inclusión se tuvieron en cuenta participantes que fueran activos físicamente por lo menos durante 1 año consecutivo; que no tuvieran restricciones médicas para el ejercicio o el desarrollo de actividad física; estudiantes universitarios que tuvieran un rango de edad entre 18 y 30 años, y que hubieran firmado el consentimiento informado.

Entre los criterios de exclusión se consideraron aquellos participantes con antecedentes médicos o alteraciones musculoesqueléticas, cardiovasculares, endocrinas y autoinmunitarias activas que restringieran la realización de actividad física. Los participantes elegidos fueron seleccionados a conveniencia.

\section{Consideraciones éticas}

Se contó con el aval del comité de ética proporcionado por la Universidad Manuela Beltrán bajo el marco del convenio Docencia - Investigación con la Universidad Santo Tomás. Cada participante fue informado respecto al objetivo del estudio y firmó el consentimiento informado considerando las pautas éticas y normativas de la Declaración de Helsinki (1961), en la cual se establece la normatividad ética y de rigor frente a los procesos investigativos en seres humanos, así como la resolución 8430 de 1993 mediante la cual se establecen las normas científicas, técnicas y administrativas para la investigación en salud en Colombia. De igual forma, se determina con base en la resolución 8430 de 1993, la cual, en el título Il, capítulo 1, el artículo 11 indica que el estudio presenta un riesgo mínimo para los participantes en los procedimientos establecidos.

\section{Procedimientos para la recolección de datos}

Para el desarrollo del estudio, el primer día se organizó una batería de pruebas que permitieron la recolección de información. Posteriormente, se realizó el diligenciamiento de datos personales y demográficos 
acompañado del Cuestionario Internacional de Actividad Física (IPAQ, International Physical Activity Questionnaire).

El nivel de actividad física fue medido a través del IPAQ en su versión corta, el cual considera tres aspectos básicos de la actividad física, que son la intensidad (caminar, moderada, vigorosa), la frecuencia (días por semana) y la duración (tiempo por día); adicionalmente, se tuvo en cuenta el tiempo que el individuo permanece sentado. EI IPAQ en su versión validada para Colombia demuestra que es un instrumento válido y confiable para determinar el estado de salud ${ }^{9}$.

Posteriormente, se calculó la actividad física semanal representada en MET, en donde caminar equivale a 3,3 MET, moderada es igual a 4 MET y vigorosa equivale a 8 MET. El cálculo de MET gastados a la semana se calculó con la siguiente fórmula: coeficiente de intensidad $\times$ número de días a la semana $\times$ número de minutos por día. Al final se suman los resultados de cada una de las categorías, MET de caminata + MET de actividad moderada + MET de actividad vigorosa, lo cual permite clasificar a los individuos según su nivel de actividad física en baja, moderada o alta ${ }^{9}$.

En las 24 horas posteriores al diligenciamiento de los cuestionarios se realizó la toma de glucometría basal, la cual fue realizada con el instrumento glucómetro de referencia OneTouch $\circledast$; los usuarios debieron presentarse en ayunas para conocer la glucemia basal de cada uno, dado que esta es una de las variables determinantes de riesgo propuesta por la International Diabetes Fedaration ${ }^{10}$. Posterior a la glucometría se realizó la medición de la composición corporal mediante bioimpedancia eléctrica con el instrumento Inbody ${ }^{\circledR}$ de referencia 770 . Es importante señalar que para que los valores obtenidos de ángulo de fase sean asertivos se deben cumplir las recomendaciones básicas, que involucran una postura adecuada frente a la máquina con abducción de piernas no mayor de $30^{\circ}$, y no existir ingesta de café, fluidos o comida al menos por 4 horas, por lo que la indicación a los usuarios fue la de mantenerse en ayunas. De igual manera, para el control del peso y el registro adecuado del agua corporal total se indicó a los participantes que hicieran vaciamiento de la vejiga y no realizaran ejercicio físico previo a la medición. Como parte adicional del control se tomó la temperatura ambiente dentro del laboratorio, con el fin de evitar pérdidas insensibles excesivas y que alteraran la información recolectada ${ }^{11}$. La información considerada como datos se relacionó con peso, análisis de obesidad, análisis de masa magra segmental, ángulo de fase, nivel de fitness y área de grasa visceral.
A las 48 horas de la evaluación de la bioimpedancia eléctrica se realizaron las mediciones de condición física, para lo que se ejecutaron las siguientes pruebas:

- Fuerza prensil manual: se aplicó el protocolo de dinamometría propuesto por la Asociación Internacional de Terapeutas de Mano; se implementó un dinamómetro análogo de mano marca Takei®. Para la ejecución de la prueba fue necesario que el sujeto se encontrara sentado con el brazo ligeramente separado del torso, el codo flexionado a $90^{\circ}$ y la muñeca neutral ${ }^{12}$. El dinamómetro debe estar ajustado al tamaño de la mano del usuario y se revisará que no presente valores diferentes de 0 que puedan alterar los resultados. La prueba dio inicio cuando el evaluador indicó al usuario que podía hacer fuerza; este debió mantener la presión por un periodo de 3 segundos. El procedimiento se realizó dos veces, tanto en el brazo dominante como en el no dominante.

- Potencia en miembros inferiores: fue evaluada mediante dos saltos de la batería de Bosco (Squat Jump y Counter Movement Jump) con el instrumento Optogait@.

La prueba de Squat Jump consistió en realizar un salto vertical con las dos extremidades inferiores a la vez, previa a la flexión mantenida de $90^{\circ}$ de las rodillas durante 5 segundos (tiempo propuesto por los evaluadores). Se buscó que el salto no presentara reutilización de energía elástica y tampoco aprovechamiento del reflejo miotático. Desde esta posición se ejecuta el salto vertical. Durante la realización del gesto, las manos deben estar en la cadera, el tronco lo más erguido posible y las piernas deben permanecer extendidas durante el tiempo de vuelo. Se realizaron dos ejecuciones, en las que se tuvo en cuenta el mejor resultado ${ }^{13}$.

En el Counter Movement Jump se parte de la posición de bipedestación con las rodillas completamente extendidas y las manos en la cadera. A la señal se realiza rápidamente una flexión de rodilla hasta completar un ángulo de $90^{\circ} \mathrm{e}$ inmediatamente se realiza el salto mediante extensión de rodilla. Se hicieron dos ejecuciones, en las que se tuvo en cuenta el mejor resultado ${ }^{14}$.

A las 72 horas de las mediciones previamente mencionadas, se llevó a cabo la evaluación de resistencia aeróbica mediante la medición indirecta del consumo máximo de oxígeno. Para ello se implementó el test de Leger, el cual consiste en una prueba de resistencia incremental audible con cambios de dirección, en 
donde el usuario deberá correr de una línea a otra (20 metros). La velocidad final alcanzada será considerada respecto a la última etapa completada, la cual permitirá estimar el $\mathrm{VO}_{2 \text { máx }}$ mediante la siguiente ecuación: $\mathrm{VO}_{2 \text { máx }}=(6 \times$ etapa final $)-27.4^{15}$.

Cabe señalar que todas las pruebas realizadas se aplicaron en horas de la mañana y cumplieron con los requerimientos metodológicos propuestos en cada uno de los protocolos de las pruebas.

\section{Análisis estadístico}

Se utilizó estadística descriptiva analítica con medidas de tendencia central para las variables escalares de naturaleza continua; posteriormente, se aplicó una prueba de normalidad de Shapiro-Wilk y se encontraron variables con distribución normal, razón por la cual las pruebas estadísticas utilizadas corresponden a pruebas paramétricas. Luego se realizó la prueba de correlación bivariada dado que fueron homogéneas con un coeficiente de Pearson con valores de significancia de $p=0.05$ con la totalidad de la población y correlaciones segmentadas en hombres y mujeres.

Se realizó una prueba de comparación de medias y se aplicó el análisis multifactorial de varianzas MANOVA; a partir de esto se propone determinar por cuartiles dónde se evidenció el fenómeno de $u$ y de $u$ invertida en las diferencias de los grupos. Nuevamente, se segmentó la población por grupos cuyos valores fuesen menores y superiores a la mediana, y otra vez se realizaron pruebas de correlación bivariada mediante prueba de Spearman. Los análisis se hicieron con el paquete estadístico SPSS ${ }^{\circledR}$ versión 25 para Windows.

\section{Resultados}

A continuación, se presentan los resultados de correlación entre cuartiles Q3-Q4; se evidenció una correlación moderada entre masa magra en tronco $(\mathrm{kg})$ $(r=0.687 ; p=0.05)$ y ángulo de fase (Fig. 1).

En Q3-Q4, se encontró correlación moderada entre tasa metabólica basal y ángulo de fase $(r=0.64$; $\mathrm{p}=0.009$ ) (Fig. 2).

Así mismo, en los cuartiles Q3 y Q4 se evidenció correlación fuerte entre nivel de fitness y ángulo de fase ( $r=0.71 ; p=0.003)$, y Counter Movement Jump (altura/cm) y ángulo de fase $(r=0.56 ; p=0.028)$ (Figs. 3 y 4).

En la comparación entre sexos se descubrió que en las mujeres existe una correlación alta entre ángulo de

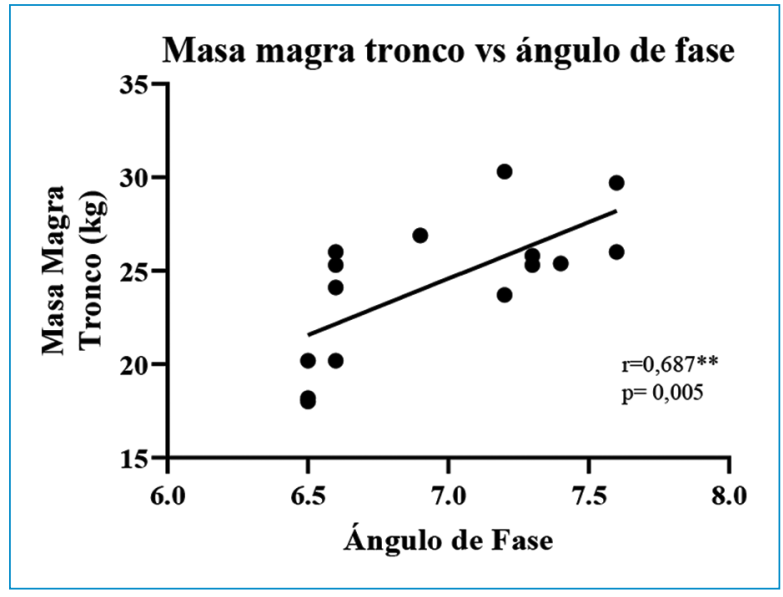

Figura 1. Relación entre la masa magra del tronco y el ángulo de fase.

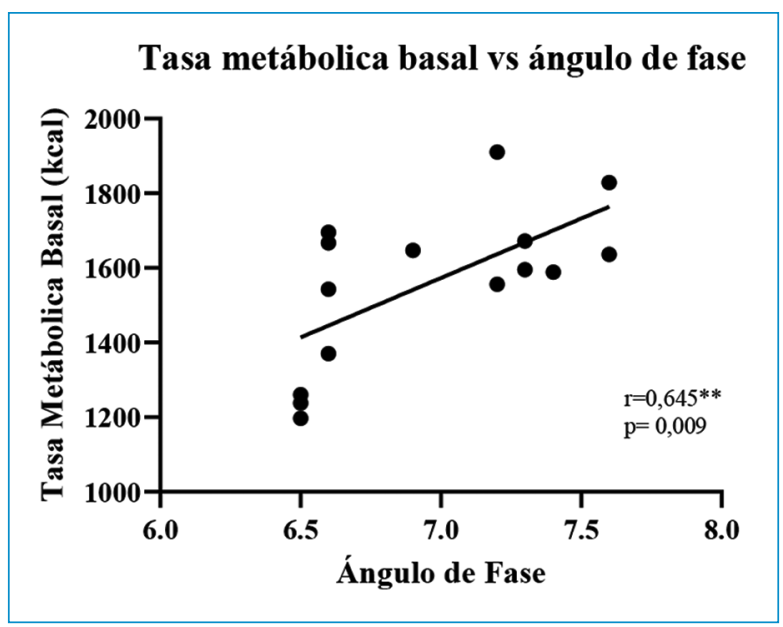

Figura 2. Relación entre la tasa metabólica basal y el ángulo de fase.

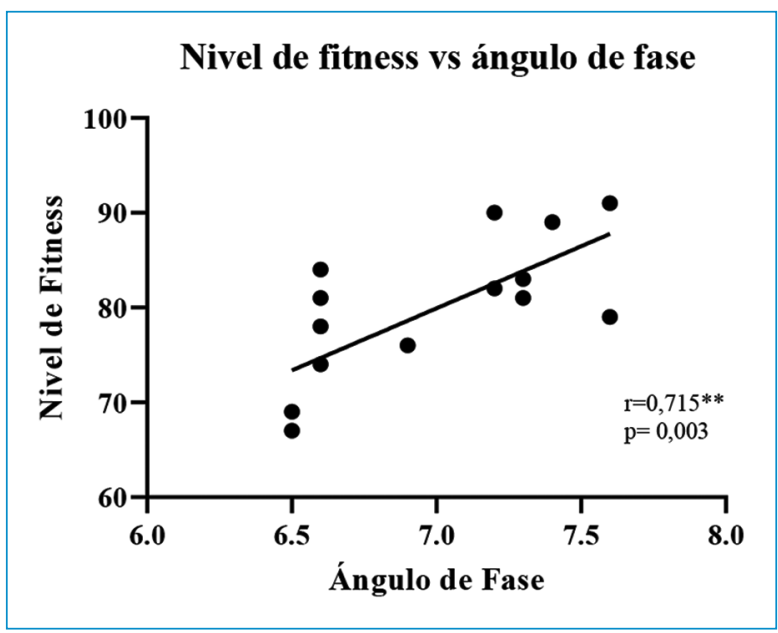

Figura 3. Relación entre el nivel de fitness y el ángulo de fase. 


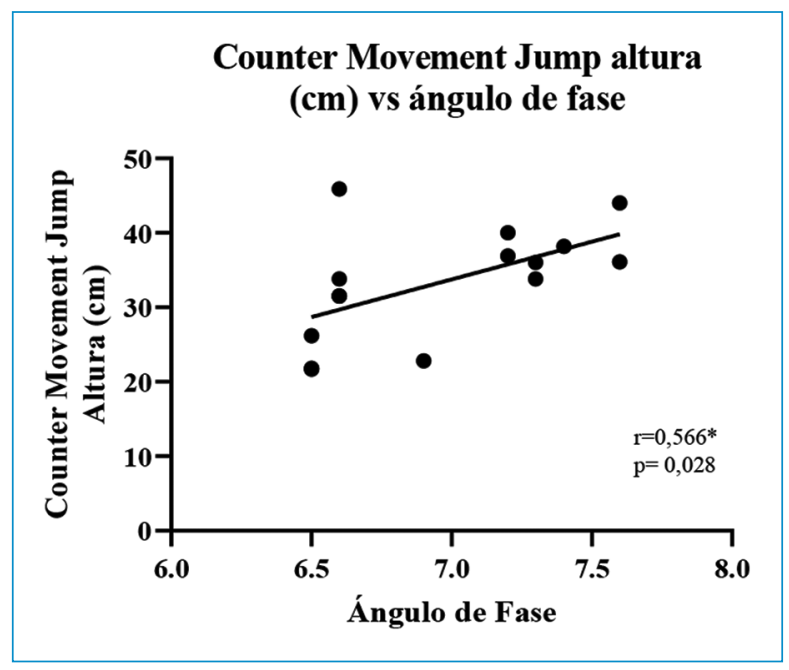

Figura 4. Relación de los resultados de la prueba de salto contra movimiento y el ángulo de fase.

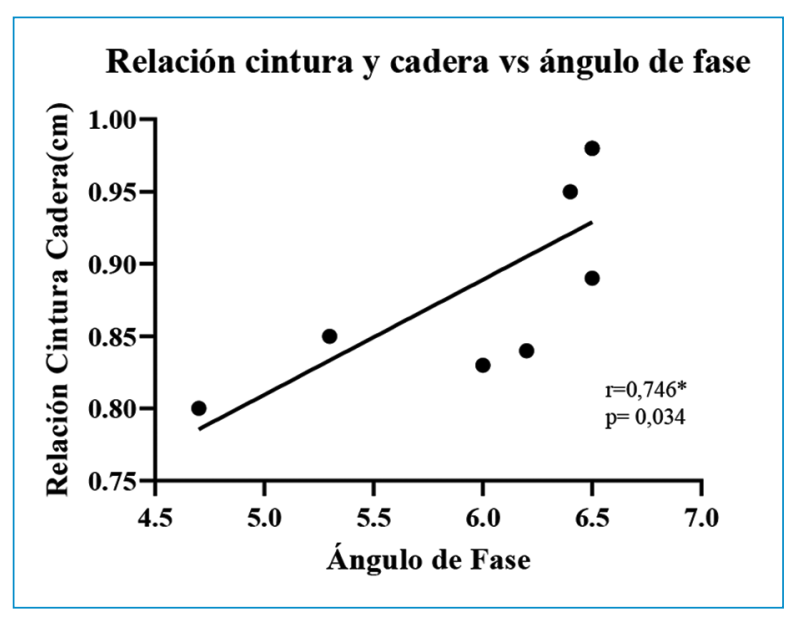

Figura 5. Relación entre el índice de cintura-cadera y el ángulo de fase.

fase e índice cintura-cadera $(r=0.74 ; p=0.034)$ (Fig. 5).

\section{Discusión}

Considerando que el objetivo inicial del presente estudio se centró en determinar la asociación entre el ángulo de fase por bioimpedancia con los indicadores de riesgo cardiovascular en población universitaria, a continuación se correlacionan los resultados obtenidos que mostraron diferencias significativas entre el ángulo de fase y variables de masa magra, fuerza prensil, fuerza explosiva evaluada a partir de las pruebas de salto, tasa metabólica basal, nivel de fitness y peso como variable de composición corporal ${ }^{16,17}$.

En cuanto a la relación entre masa magra y ángulo de fase, se ha podido determinar, en primera instancia, que existen diferencias anatómicas y funcionales importantes respecto a la distribución muscular y de tejido adiposo entre hombres y mujeres, lo cual ha sido demostrado en distintas investigaciones que evidencian que la distribución regional del tejido adiposo es el principal factor que expone la correspondencia entre adiposidad y riesgo cardiometabólico ${ }^{18,19}$. Tales diferencias están determinadas genéticamente y son evidentes cuando se evalúan la amplitud pélvica y su relación con la columna lumbosacra, coxofemoral y los miembros inferiores; estas regiones corporales, en el caso de las mujeres, son más amplias y con ciertos grupos musculares, como los estabilizadores del tronco, que no son tan activos comparados con los varones, por lo que se podría inferir que zonas como el tronco, la cadera y las piernas se convierten fácilmente en reservorios de grasa corporal, por lo que el ángulo de fase tendería a reducirse, incrementando potencialmente el riesgo cardiovascular en las mujeres ${ }^{20,21}$. Es por ello que la activación muscular y el fortalecimiento de estas regiones dependerá de manera importante del trabajo muscular combinado del abdomen, la cadera y las piernas ${ }^{22}$.

Por otra parte, se observó que para ambos sexos hubo una correlación moderada entre el ángulo de fase y la masa magra en los miembros superiores, lo cual está altamente relacionado con la fuerza prensil de los sujetos. Si bien es cierto que la fuerza prensil ha sido considerada como una de las variables de condición física que predice, en cierta medida, el riesgo potencial de padecer enfermedades cardiovasculares, algunos estudios refieren que la población juvenil que presente valores superiores al percentil 50 tienen un menor riesgo de padecer enfermedades cardiovasculares ${ }^{23,24}$.

A partir de lo anterior, es posible establecer la existencia de una relación directa y significativa entre la fuerza muscular del tren superior y los indicadores de riesgo cardiovascular, entendiendo que el tejido muscular se convierte en un factor protector que determina un perfil más saludable. De acuerdo con lo anterior, el incremento del tejido muscular reduce las posibilidades de enfermedades cardiovasculares puesto que, aparte de su función motora, desempeña funciones endocrinas que regulan efectivamente los niveles de glucosa y el metabolismo de las grasas, entre otras funciones hormonales, lo cual demuestra que en población 
juvenil, cuando hay valores superiores en fuerza prensil por dinamometría, existe menor incidencia de factores de riesgo cardiovascular ${ }^{25}$.

Considerando la relación existente entre el ángulo de fase y la fuerza explosiva, se logra demostrar que en aquellos participantes cuya fuerza explosiva fue menor se obtuvo un valor de ángulo de fase reducido, por lo que es necesario recalcar que una depleción sobre esta variable aun en población joven puede estar condicionada a cambios en el sistema muscular, los cuales pueden estar asociados con el detrimento de fibras rápidas (fast twich) y el incremento en el porcentaje de fibras lentas (slow twich), lo cual induce de manera concomitante a alteraciones e imbalances en los patrones de coordinación intra-intermuscular ${ }^{26,27}$, asociados a variaciones en las concentraciones hormonales entre cortisol, testosterona y hormona de crecimiento, a variaciones en el potencial electroquímico del músculo esquelético y a muerte de motoneuronas alfa. Si bien esto es cierto, considerar estos aspectos y aunarlos con malos hábitos de vida puede incrementar el riesgo de padecer en la adultez enfermedades cardiovasculares $^{28}$.

En cuanto a la relación que existe entre el ángulo de fase y la tasa metabólica basal de los participantes, se puede determinar que el incremento de esta demuestra un consumo mayor de kilocalorías, lo cual es un reflejo de la realización de actividad física regular en los sujetos. Pese a que la correlación se evidenció más en el sexo masculino, es importante comprender que el incremento de esta variable se asocia con la proporción de masa muscular, las variaciones en las concentraciones hormonales, la superficie corporal y la edad, entre otros; no obstante, una tasa metabólica basal alta es un indicativo que sugiere una menor probabilidad de padecer enfermedades cardiovasculares, razón por la cual, al evaluar el ángulo de fase, la reactancia cambia y este tiende a ser mayor ${ }^{29,30}$.

Ahora bien, al comparar los datos obtenidos por bioimpedancia en concordancia con otros métodos de referencia, se encuentra que esta báscula fue validada con el método DXA por Ling, et al. ${ }^{31}$, en cuyo estudio compararon los resultados de la masa magra en población normal y con sobrepeso, encontrando una correlación del $99 \%$. Por su parte, Miller et al. ${ }^{32}$ compararon los resultados del porcentaje graso de los dos métodos (Inbody y DXA) y hallaron una relación significativa $(r=0.94 ; p<0.0001)$. Donadio, et al.$^{33}$, en otro estudio realizado con pacientes renales crónicos, encontraron una buena correlación entre los valores obtenidos de ángulo de fase y los arrojados por métodos como el DXA cuando analizaron la composición corporal.

Finalmente, la asociación entre ángulo de fase y nivel de fitness es un indicativo de la salud física que es conexo con hábitos y estilos de vida saludable que implican la práctica regular de actividad física y una nutrición sana. Estos aspectos favorecen el reclutamiento muscular, con la subsecuente ganancia de masa magra y de fuerza muscular, los cuales se asocian con la adecuada celularidad, la integridad de las membranas celulares y el balance hídrico ${ }^{34,35}$.

El diseño del presente estudio puede considerarse como una limitación para la determinación de causalidad. Por consiguiente, no es posible asegurar que el ángulo de fase sea una variable determinante para la evaluación del riesgo cardiovascular. En forma similar, estos hallazgos solo son evidentes en un grupo de sujetos de la muestra total Q3 a Q4 con indicadores indirectos de riesgo cardiovascular. Sin embargo, el estudio muestra relaciones entre las variables que pueden sugerir el ángulo de fase como un buen indicador para estimar el riesgo cardiovascular ${ }^{6,8,30,34}$, teniendo en cuenta que los indicadores utilizados son empleados para la evaluación de este riesgo en diversos estudios $^{35,36}$. Finalmente, se recomienda para futuros estudios obtener muestras representativas para evaluar esta relación.

\section{Conclusiones}

La bioimpedancia es una herramienta útil y actualmente se convierte en un elemento de utilidad no solo para determinar la composición corporal, sino también para tener una visión más exacta de la predisposición y la potencialidad de padecer enfermedad cardiovascular, puesto que permite analizar distintas variables de forma precisa y en todos los segmentos corporales, discriminar elementos óseos, musculares, grasos y agua, entre otros, y complementar de manera óptima las evaluaciones de la composición corporal convencionales cuyo fin es aproximarse a un diagnóstico más confiable y asertivo.

El ángulo de fase como variable independiente de la bioimpedancia se convierte en un elemento predictor de riesgo cardiovascular en la población participante, puesto que a partir de elementos básicos y sencillos, como el análisis de la resistencia y la reactancia de los tejidos corporales, permite comprender estados nutricionales y de composición corporal propios de un sujeto; este se puede emplear en otras poblaciones en las que sea necesario realizar este tipo de análisis de 
manera más precisa. Pese a que para este caso se analizó población universitaria, es importante resaltar los cambios sutiles encontrados, en especial en las mujeres; no obstante, se sugiere la realización de más estudios con muestras más amplias y con variación de la edad en la población participante.

\section{Financiamiento}

Este estudio fue financiado por la Facultad de Cultura Física, Deporte y Recreación, Universidad Santo Tomás, Bogotá, Colombia.

\section{Conflicto de intereses}

Los autores del presente proyecto declaran que no presentan ningún conflicto con personas, compañías o instituciones que pudiera dar lugar a intereses en relación con el artículo que se remite para publicación.

\section{Responsabilidades éticas}

Protección de personas y animales. Los autores declaran que los procedimientos seguidos se conformaron a las normas éticas del comité de experimentación humana responsable y de acuerdo con la Asociación Médica Mundial y la Declaración de Helsinki.

Confidencialidad de los datos. Los autores declaran que han seguido los protocolos de su centro de trabajo sobre la publicación de datos de pacientes.

Derecho a la privacidad y consentimiento informado. Los autores han obtenido el consentimiento informado de los pacientes y/o sujetos referidos en el artículo. Este documento obra en poder del autor de correspondencia.

\section{Bibliografía}

1. Cosi G, Reátegui M. Riesgo cardiovascular según score Framingham de las enfermeras de un instituto nacional; agosto - noviembre. [dissertation]. Repositorio Universidad Peruana Cayetano Heredia; 2018. [Citado 12 de febrero de 2020]. 32 p. Disponible en: http://repositorio.upch.edu. pe/bitstream/handle/upch/4350/Riesgo_CosiCano_Gabriela.pdf?sequen$\mathrm{ce}=1$ \&isAllowed $=\mathrm{y}$.

2. Cruz A, Correas L, Ronconi M, Fernández R, Porta J. La bioimpedancia eléctrica como método de estimación de la composición corporal: normas prácticas de utilización. Rev Andal Med Deporte. 2011;4:167-74.

3. Quesada L, León C, Betancourt J, Nicolau E. Elementos teóricos y prácticos sobre la bioimpedancia eléctrica en salud. Rev Arch Med Camagüey. 2026;20:565-78.

4. Sánchez A, Fernández M, Teruel J. Fundamentos eléctricos de la bioimpedancia. Nefrología. 2012;32:133-5.

5. Rondón A, Vallejo F, García M. Evaluación nutricional mediante técnicas de impedancia. Ventajas e inconvenientes en TCA [dissertation]. Repositorio Universidad Pablo de Olavide; 2014. [Citado 12 de febrero de 2020]; p. 2090-114. Disponible en: http://www.tcasevilla.com/archivos/ impedancia_bioelectrica_en_tca.pdf.
6. Saad M, Jorge J, Martins W, Cardoso G, Santos M, Rosa M, et al. Phase angle measured by electrical bioimpedance and global cardiovascular risk in older adults. Geriatr Gerontol Int. 2018;18:732-7.

7. Llames L, Baldomero V, Iglesias M, Rodota L. Valores del ángulo de fase por bioimpedancia eléctrica; estado nutricional y valor pronóstico. Nutr Hosp. 2013;28:286-95.

8. Vellido V. Relación del ángulo de fase determinado por bioimpedanciometría con factores de riesgo cardiovascular, adipocitoquinas, antropometría e ingesta dietética de pacientes obesos [dissertation]. Repositorio Universidade da Coruña; 2014. [Citado 12 de febrero de 2020]. 359 p. Disponible en: https://ruc.udc.es/dspace/bitstream/handle/2183/13949/ BellidoCastaneda_Virginia_TD_2015.pdf.

9. Jiménez J, Obregón M, Tamayo G. Medición de la actividad física IPAQ corto versión Colombia de los estudiantes de primer semestre de la Universidad San Buenaventura Medellín. Repositorio Universidad San Buenaventura. 2019. [Citado 25 de diciembre de 2019]. p. 1-21. Disponible en: http://45.5.172.45/bitstream/10819/7337/1/Medicion_Actividad_ Fisica_Jimenez_2019.pdf

10. Carvalho V, Bressan J, Babio N, Salas S. Prevalence of metabolic syndrome in Brazilian adults: a systematic review. BMC Public Health. 2013;13:1-10.

11. Alvero J, Correas L, Ronconi M, Fernández R, Porta IJ. La bioimpedancia eléctrica como método de estimación de la composición corporal: normas prácticas de utilización. Rev Andal Med Deporte. 2011;4: 167-74.

12. González CH, Sepúlveda LE, Santafé LR. Different reference values for hand dynamometry: a conflicting issue. Nutr Clin Dit Hosp. 2017;37: 104-10.

13. Villa J, García J. Tests de salto vertical (I): aspectos funcionales. Revista Digital: Rendimiento Deportivo.com. 2003;6:1-14. [Citado el 26 de diciembre de 2019]. Disponible en: https://www.researchgate.net/publication/301960181_Tests_de_salto_vertical_I_Aspectos_funcionales.

14. Vélez M. El entrenamiento de fuerza para la mejora del salto. Apunts de Medicina de l'Esport. 1992;29:139-56. [Citado el 26 de diciembre de 2019]. Disponible en: https://www.apunts.org/es-el-entrenamiento-fuerza-mejora-del-articulo-X0213371792052916.

15. García C, Secchi D. Test Course Navette de 20 metros con etapas de un minuto. Una idea original que perdura hace 30 años. Apunts Medicina de l'Esport. 2014;49:93-103. [Citado el 26 de diciembre de 2019]. Disponible en: https://www.apunts.org/es-test-course-navette-20metros-con-articulo-X0213371714492019.

16. Villa J, García J. Tests de salto vertical (I): aspectos funcionales. Revista Digital: Rendimiento Deportivo.com. 2003;6:1-14. [Citado el 26 de diciembre de 2019]. Disponible en: https://www.researchgate.net/publication/301960181_Tests_de_salto_vertical_I_Aspectos_funcionales.

17. Battaglia G, Paoli A, Bellafiore M, Bianco A, Palma A. Influence of a sport-specific training background on vertical jumping and throwing performance in young female basketball and volleyball players. J Sports Med Phys Fitness. 2014:54:581-7.

18. Shorr M, Dichtel L, Gerweck A, Valera R, Torriani M, Miller K, et al. Sex differences in body composition and association with cardiometabolic risk. Biol Sex Differ. 2018;9:28.

19. Bruening DA, Frimenko RE, Goodyear CD, Bowden DR, Fullenkamp AM. Sex differences in whole body gait kinematics at preferred speeds. Rev Gait Posture. 2015;41:540-5.

20. Nindl BC, Harman EA, Marx JO, Gotshalk LA, Frykman PN, Lammi E, et al. Regional body composition changes in women after 6 months of periodized physical training. Rev J Appl Physiol. 2006;88:2251-9.

21. Ko YS, You SE. Comparisons of physical fitness and body composition among Sasang types with and without body mass index as a covariate. Rev Integr Med Res. 2015;4:41-7.

22. Rodríguez-Rodríguez F, Santibáñez-Miranda M, Montupin-Rozas G, Chávez-Ramírez F, Solis-Urra P. Diferencias en la composición corporal y actividad física en estudiantes universitarios según año de ingreso. Rev Univ Salud. 2016;18:474-81.

23. Ruiz JR, Ortega FB, Wärnberg J, Moreno LA, Carrero JJ, Gonzalez-Gross M, et al. Inflammatory proteins and muscle strength in adolescents: The Avena Study. Rev Arch Pediatr Adolesc Med. 2008;162: 462-8.

24. Schlussel MM, dos Anjos LA, de Vasconcellos MT, Kac G. Reference values of handgrip dynamometry of healthy adults: a population-based study. Rev Clin Nutr. 2008;27:601-7.

25. Silventoinen K, Magnusson PK, Tynelius P, Batty GD, Rasmussen F. Association of body size and muscle strength with incidence of coronary heart disease and cerebrovascular diseases: a population-based cohort study of one million Swedish men. Rev Int $\mathrm{J}$ Epidemiol. 2009;38:110-8.

26. Ramírez JF, León HH. Características antropométricas, funcionales y de fuerza explosiva de mujeres mayores de 50 años físicamente activas de la ciudad de Bogotá, Colombia. Revista Esp Ger Gerontol. 2012;47: 148-54. 
Rev Colomb Cardiol. 2021;28(6)

27. Pereira Rodríguez JE, Velásquez-Badillo X, Santamaría-Perez KN, Figueroa-Tiburcio MA, Avendaño-Aguilar JA, Marin-Herrera L, et al. Relación entre la fuerza prensil y los marcadores de riesgo cardiovascular en jóvenes universitarios. Cienc Salud Virtual. 2019;11:90-101. [Citado el 3 de febrero de 2021]. Disponible en: https://revistas.curn.edu.co/index. $\mathrm{php} /$ cienciaysalud/article/view/1325

28. Vargas M, Lancheros L, Barrera M. Gasto energético en reposo y composición corporal en adultos. Rev Fac Med. 2011;59:43-58

29. Bryce-Moncloa A, Alegría-Valdivia E, San Martín-San Martín MG. Obesidad y riesgo de enfermedad cardiovascular. An Fac Med. 2017;78: 202-6.

30. Bioelectrical impedance analysis in body composition measurement: $\mathrm{Na}$ tional Institutes of Health Technology Assessment Conference, Statement. Am J Clin Nutr. 1996;64(Suppl):524S-32S.

31. Ling $\mathrm{CH}$, de Craen AJ, Slagboom PE, Gunn DA, Stokkel MP, Westendorp RG, et al. Accuracy of direct segmental multi-frequency bioimpedance analysis in the assessment of total body and segmental body composition in middle-aged adult population. Rev Clin Nutr. 2011;30:610-5.
32. Miller R, Chambers T, Burns S. Validating InBody® 570 multi-frequency bioelectrical impedance analyzer versus DXA for body fat percentage analysis. Rev Journal of Exercise Physiology Online. 2016;19:71-8. [Citado el 15 de julio de 2020]. Disponible en: https://www.asep.org/asep/ asep/JEPonlineOCTOBER2016_Miller.pdf.

33. Donadio C, Halim AB, Caprio F, Grassi G, Khedr B, Mazzantini M. Single- and multi-frequency bioelectrical impedance analyses to analyse body composition in maintenance haemodialysis patients: comparison with dual-energy x-ray absorptiometry. Rev Physiol Meas. 2008;29:S517-24.

34. Abad S, Sotomayor G, Vega A, Pérez de José A, Verdalles U, Jofré R, et al. The phase angle of the electrical impedance is a predictor of long-term survival in dialysis patients. Nefrologia. 2011;31: 670-6.

35. Barbosa-Silva MC, Barros AJ, Wang J, Heymsfield SB, Pierson RN Jr. Bioelectrical impedance analysis: population reference values for phase angle by age and sex. Rev Am J Clin Nutr. 2005;82:49-52.

36. Bottaro F. Diseño de los estudios de investigación. Debilidades y fortalezas. Hematologia. 2014;18:74-83. 